\title{
Sensitivity of identifying cut-off lows in the Southern Hemisphere using multiple criteria: implications for numbers, seasonality and intensity
}

\author{
Henri Rossi Pinheiro ${ }^{1}\left[\right.$ ] Kevin Ivan Hodges ${ }^{2} \cdot$ Manoel Alonso Gan $^{1}$ \\ Received: 16 May 2019 / Accepted: 22 August 2019 / Published online: 14 September 2019 \\ (c) The Author(s) 2019
}

\begin{abstract}
Cut-off Low (COLs) are often associated with heavy precipitation and strong wind events, but there are still uncertainties on how their identification affect the numbers and seasonality. This paper aims to determine the sensitivity of identifying Southern Hemisphere COLs in the ERA-Interim reanalysis to different types of identification criteria. Upper-level cyclones are initially tracked on the $300 \mathrm{hPa}$ level using relative vorticity and geopotential in order to present different perspectives. This reveals significant differences between the numbers and length of the identified tracks for the two fields. To identify the COLs different post-tracking filters are applied which are divided into two steps. Firstly, three filters are considered to separate cut-off lows from open troughs by sampling winds at different offset radial distances from the upper-level cyclonic centres. Secondly, potential vorticity and temperature criteria are imposed to determine how these conditions affect the identified COLs in terms of numbers, seasonality and intensity. It was found that methods based on multiple criteria restrict the COL identification by imposing specific characteristics, while methods based on simpler schemes (e.g. using only winds) can detect larger samples of COLs observed visually in the geopotential maps. Although it is difficult to say which method is more accurate in identifying COLs, because of the subjective aspect of observer's assessment, a scheme using only winds should be more representative of reality as this simply imposes on the detection system a cyclonic circulation appearance regardless of the physical and dynamical characteristics. Therefore, this type of method could be considered as a standard method for identifying COLs that can be used for either operational or research purposes.
\end{abstract}

Keywords Cut-off lows $\cdot$ Objective methods $\cdot$ Identification $\cdot$ Criteria $\cdot$ Uncertainties

\section{Introduction}

Cut-off Lows (COLs) are synoptic-scale low pressure systems that form at upper levels and can be identified as minima in geopotential charts. The historical interest in COLs is motivated by their importance to the local weather as they are often responsible for heavy precipitation and floods in different regions of the world (Singleton and Reason 2006; Llasat et al. 2007; McInnes and Hubbert 2001). COLs also play an important role in stratosphere-troposphere exchange, where air and atmospheric constituents are transported across the tropopause, and occasionally lead to episodes of high ozone concentration in the lower troposphere (Ancellet et al. 1994; Rondanelli et al. 2002). Over past years, there have been great efforts to have a better understanding of observed COLs with several studies focusing on their climatological aspects. 
The earliest climatologies of COLs were performed for particular regions based on relatively old datasets and manual methods using visual inspection of available charts and satellite images (Taljaard 1985; Price and Vaughan 1992; Kentarchos and Davis 1998). The drawback in using manual methods is that they rely on subjective decisions based on human expertise, requiring an exhaustive analysis for long periods of data. The advances and improvements in observations and computer systems (e.g. more modern reanalysis products and automated techniques for atmospheric system detection) have allowed statistics of COLs to be reproduced fairly consistently over time by using larger samples of data and larger domains than used in earlier studies based on subjective analysis.

Developing objective methods for detecting and tracking atmospheric systems represents fundamental aspects of assessment and intercomparison between reanalyses or model outputs. However, a problem concerning the use of objective approaches is the variety of methods used to detect and track COLs. The idea of considering a unique method to identify COLs and providing consistent results is particularly important, but difficult to realise because of the different criteria often chosen to define the COL features motivated by different conceptual ideas of what a COL is. One of the more subtle points in COL identification is the method by which a COL is defined. Objective methods need to have an identifiable physical basis with specific constraints in order to yield consistent results.

A wide range of techniques have previously been used as a means of identifying COLs or mid-upper level tropospheric systems. These methods differ in the way features are detected and tracked in consecutive frames of data. COLs are typically analysed using local extremes of geopotential or vorticity with additional criteria introduced to determine the cut-off condition, though COLs can also be defined based on a conceptual model in terms of cold core, high potential vorticity (PV) anomaly, and baroclinic zones. Probably the most widely used objective method for COL identification is that developed by Nieto et al. (2005), which is based on the following steps: geopotential minimum, cut-off circulation, thermal frontal parameter, and equivalent thickness. The latter in particular is used to search for a thickness ridge east of the COL centre or, in other words, the cold core criterion, which is applied in the layer 200-300 hPa. Similar methods adapted for the Southern Hemisphere ( $\mathrm{SH}$ ) were used by Reboita et al. (2010) and Ndarana and Waugh (2010) with some differences in the level or layer used to compute the parameters. A particularly intriguing aspect of these differences is the different levels used to detect cold cores and how this choice affects the results. The differences in the methodologies certainly lead to discrepancies between studies, introducing uncertainties between the studies and their conclusions.
There are a number of studies describing different types of methods for the identification of COLs using automatic (Nieto et al. 2005; Ndarana and Waugh 2010; Favre et al. 2012; Pinheiro et al. 2017) or semi-automatic methods (Fuenzalida et al. 2005; Campetella and Possia 2007). Objective schemes appear more accurate in detecting COLs when using several parameters. To identify the COL features, some methods use PV as a stratospheric tracer (Wernli and Sprenger 2007; Ndarana and Waugh 2010) since the process of COL genesis is accompanied by tropopause folding and pronounced intrusions of anomalous cyclonic PV tongues, eventually leading to Rossby wave breaking (RWB) as discussed in Hoskins et al. (1985). More recently, Pinheiro et al. (2017) used a method to identify COLs that combines the search for cold core with high cyclonic PV. These authors suggest that the use of a scheme based on multiple criteria allows specific features to be imposed to the selected sample, though this may reduce dramatically the number of detected systems.

A comparison between two different objective methods was presented by Nieto et al. (2008) for COLs in the Northern Hemisphere. One approach is based on a conceptual model proposed by Nieto et al. (2005) while the other uses PV to follow stratospheric and tropospheric cutoffs (Wernli and Sprenger 2007). The results show large differences in numbers and spatial distribution between the two identification methods, which can be attributed to how each method counts the features per unit area as well as the level where the COLs are identified. For the latter, the authors suggest that the more appropriate level to identify COLs depends on region and season since lower (higher) isentropic levels represent better the COLs occurring at higher (lower) latitudes.

The location of the main areas of COL activity in the $\mathrm{SH}$ are generally consistent in the literature, but the numbers and seasonality differ considerably between studies. Therefore, one research question arises as to whether the discrepancies between studies are mainly due to uncertainties in using different reanalyses, as discussed in the recent study of Pinheiro et al. (2019), or whether the method used to identify COLs also has important implications for the results. In relation to the latter, large differences were found between the statistics produced by a tracking algorithm applied to vorticity and geopotential fields, since these focus on different spatial scale features (Hoskins and Hodges 2005).

Since COLs are important for impacts such as heavy precipitation and strong winds, identifying COLs correctly may be helpful both in numerical weather prediction (NWP) and climate models. Thus, this study was motivated by attempts to understand how sensitive the identification of SH COLs are to different methods and schemes. The goal of this study is to examine the sensitivity of identifying COLs to the field used for tracking (relative vorticity or geopotential) and to the different filters used to separate COLs from 
other systems, that make use of simple schemes (using only horizontal winds) and multiple step schemes (using also temperature and/or PV). Hence, we intend to verify how the choice of method used to identify COLs affects their numbers, seasonality and intensity, attempting to demonstrate which method/criteria are the most reproducible and reliable for COL identification, which may be considered as a single standard method to be adopted universally.

\section{Reanalysis dataset}

The data used in this study are obtained from the ERAInterim (ERAI) reanalysis (Simmons et al. 2007), produced by the European Centre for Medium Range Weather Forecast (ECMWF). The six-hourly data are produced with a fourdimensional variational data assimilation (4D-Var) system. The reanalysis is performed using a spectral model using a N128 reduced Gaussian grid (corresponding to approximately $79 \mathrm{~km}$ or $0.75^{\circ}$ ) and 60 vertical hybrid levels. ERAI has an improved atmospheric model and data assimilation system with variational bias correction for satellite radiances compared to the older ECMWF 40-year Reanalysis (ERA-40 reanalysis), which minimizes inconsistencies in the observations (Dee et al. 2011). Sea surface temperature (SST) and sea ice concentration (SIC) are prescribed boundary conditions for the atmospheric forecast model. ERAI was used for a 36-year period (1979-2014) in this study.

\section{Tracking and COL identification}

In this study we used the TRACK algorithm (Hodges 1994, $1995,1999)$ to track features of interest. The full process for tracking features of interest in the chosen fields is performed objectively in three main stages, which are: pre-processing filtering, tracking, and post-tracking filtering. Initially the large-scale background is removed by performing a spherical harmonic decomposition of the fields and setting the coefficients of the total wavenumbers less than or equal to five to zero. For the $300-\mathrm{hPa}$ geopotential $\left(Z_{300}\right)$ the zonal mean is first removed from the data, i.e., each grid point is subtracted from its mean latitudinal value. The use of the zonal anomaly of geopotential $\left(Z_{300}^{\prime}\right)$ for the tracking allows the "weak" extremes to be more easily identified than in the raw geopotential, particularly at lower latitudes where the geopotential gradient is typically weaker than at higher latitudes.

The tracking of SH COLs is performed using six hourly data based on the $\xi_{300}$ and $Z_{300}^{\prime}$ minima in order to present different perspectives. The data are also spectrally truncated to triangular truncation 42 (T42) on a Gaussian grid for relative vorticity, as this is a very noisy field, whilst T63 is used for the geopotential field, similar to previous studies (Pinheiro et al. 2017, 2019). The filtering focuses the identification on the same spatial scale for each data set which allows a fairer comparison between data with different resolutions. In addition to the truncation, a spectral tapering is applied to reduce the Gibbs phenomena in exactly the same way as done in Hoskins and Sardeshmukh (1984).

Candidate features are first identified as minima below chosen thresholds for each field, $-1.0 \times 10^{-5} \mathrm{~s}^{-1}$ for $\xi_{300}$ and -50 geopotential meters (gpm) for $Z_{300}^{\prime}$, where the minus sign for relative vorticity is required for cyclonic systems in the SH. Results indicate that the number of tracks obtained are sensitive to these thresholds. In general, increasing the magnitude of these thresholds (making them more negative) reduces the lifetime and the number of COLs detected. The use of the weak negative values for the tracking step is appropriate to identify a large number of potential COLs, which will be filtered further in the post-tracking step in order to exclude upper-level troughs. If we set a large threshold for the tracking, weak COLs will be missed in the analysis.

The tracking is performed by first initialising a set of tracks from the feature points using the nearest neighbour approach, these tracks are then refined by minimising a cost function for track smoothness subject to adaptive constraints (Hodges 1999). The tracking is the same as used in Pinheiro et al. (2017) but with some modifications to the adaptive tracking constraints for displacement distance and smoothness. These are determined taking into account the type of motion for the observed systems of interest, supported by a limited sensitivity study which showed the different possible matches for the COL tracks. A description of the adaptive constraints used in this study and their differences from those applied in Pinheiro et al. (2017) are presented in the Appendix. Following completion of the tracking the tracks are filtered to retain those that last longer than $24 \mathrm{~h}$ in order to exclude very short lifecycles, i.e. to eliminate or filter an unwanted type of motion (Holton 1992).

In order to identify the COLs from among all the tracked features additional field information is added to the tracks with the aim of detecting a cut-off circulation. This is done by referencing the $300-\mathrm{hPa}$ horizontal wind components $\left(U_{300}, V_{300}\right)$ to the tracks at a fixed radial distance of $5^{\circ}$ (geodesic distance) from the COL centre in several directions relative to the centre. Similar filters have been previously used as a means of identifying upper tropospheric vortices (Costa 2009; Morais 2016; Nieto et al. 2005). The three types of filters used to detect the possible cut-off low formation are shown in Fig. 1 and described as follows:

- Single-point filter: uses a single sample of $U_{300}$ at a single offset point (similar to Nieto's algorithm) located at $180^{\circ}$ relative to North (Fig. 1a) where the radial dis- 
Fig. 1 Two-dimensional schematic representation of the 300-hPa horizontal winds in the identification of Southern Hemisphere Cut-off Lows using the a single-point filter, $\mathbf{b}$ fourpoints filter, and $\mathbf{c}$ eight-points filter. The centre of the spherical cap represents the $\xi_{300}$ or $Z_{300}^{\prime}$ minimum. Positive and negative values determine the direction of the 300-hPa horizontal wind components $(\mathrm{u}, \mathrm{v})$ (a) single-point filter

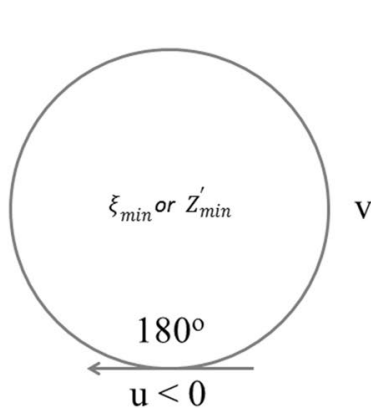

(b) four-point filter

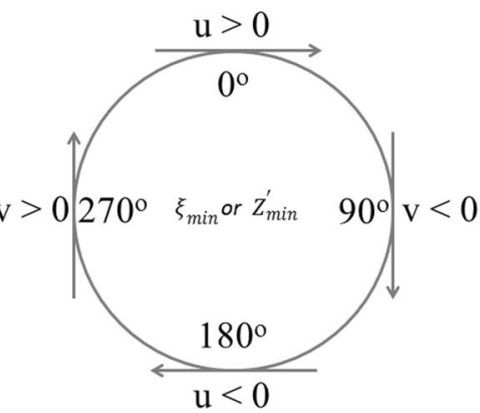

(c) eight-point filter

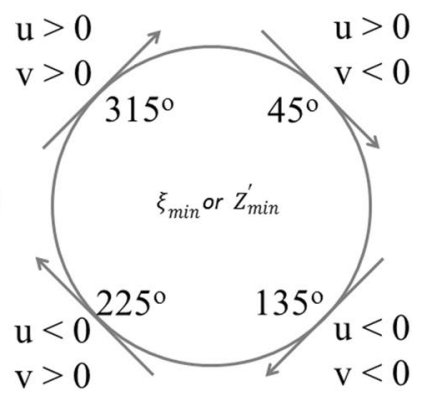

tance is $5^{\circ}$ from the $\xi_{300}$ or $Z_{300}^{\prime}$ minima. If the sample value is negative (easterly direction) for four consecutive steps ( 1 day), this track is considered a potential COL.

- Four-point filter: uses two samples for each $U_{300}$ and $V_{300}$ at the four different offset points of $0^{\circ}\left(U_{300}>0\right)$, $90^{\circ}\left(V_{300}<0\right), 180^{\circ}\left(U_{300}<0\right)$, and $270^{\circ}\left(V_{300}>0\right)$ relative to North (Fig. 1b) at the radial distance of $5^{\circ}$ from the $\xi_{300}$ or $Z_{300}$ minima.

- Eight-point filter: uses four samples for each $U_{300}$ and $V_{300}$ at the four different offset points of $45^{\circ}\left(U_{300}>0\right.$, $\left.V_{300}<0\right), 135^{\circ}\left(U_{300}<0, V_{300}<0\right), 225^{\circ}\left(U_{300}<0\right.$, $\left.V_{300}>0\right)$, and $315^{\circ}\left(U_{300}>0, V_{300}>0\right)$ relative to the North (Fig. 1c) at the radial distance is $5^{\circ}$ from the $\xi_{300}$ or $Z_{300}$ minima.

As well as the wind identification criteria the impact of using multiple criteria schemes is investigated here. This is done by adding further variables one-by-one for temperature and PV in order to identify the cold-core and stratospheric intrusion conditions, respectively. These are the most often used criteria for identifying COLs, and the sensitivity analysis allows us to verify how temperature and PV as well as their combination affects the COL identification. The full set of criteria is exactly the same as used in Pinheiro et al. (2017), where the COL tracks are referenced to zonal anomalies of the 300-hPa PV $\left(P V_{300}\right)$ and the $300-\mathrm{hPa}$ temperature $\left(T_{300}\right)$ by searching for the minimum value over a spherical cap region of $5^{\circ}$ centered on the $\xi_{300}$ minima. Tracks with $T_{300}$ values lower than -3 Kelvin and $P V_{300}$ values lower than -2 PVU (1 PVU $=10^{-6} \mathrm{~m}^{2} \mathrm{~s}^{-1} \mathrm{~K} \mathrm{~kg}^{-1}$ ) for at least four consecutive steps ( 1 day) are retained. Additionally, an easterly wind (defined for the zonal wind values lower than $-8 \mathrm{~m} / \mathrm{s}$ ) must occur at $5^{\circ}$ south from the $\xi_{300}$ minima. A potential COL is defined using a multiple step scheme when the steps described above are satisfied. These criteria were applied independently to the wind filter described above in order to verify the sensitivity of different choices of either of the three parameters $\left(U_{300}, P V_{300}\right.$ and $\left.T_{300}\right)$ with respect to the $\mathrm{COL}$ identification. It is possible to examine the effect of $P V_{300}$ and $T_{300}$ together with the wind criteria, but this would not change the conclusions.

Additionally, COL tracks obtained using the different methods are individually compared with each other through a track matching algorithm (Hodges et al. 2011), which is used to match the identically same COLs with mean separation distance less than $4^{\circ}$ (geodesic) that overlap in time by at least $50 \%$ of the track points. Spatial statistics for the identified COLs are computed using spherical kernel estimators (Hodges 1996).

Since there is wide variation in the definition of COLs, a standard definition of COLs needs to be established in order to facilitate the method assessments. This study uses the classical definition of Palmén (1949) for COLs referring to those cyclonic circulation systems in the upper troposphere that are "cut-off" from the westerlies (i.e. a definition that is independent of the physical and dynamical characteristics). To avoid the numerous higher latitude vortices, only the observed COLs located north of $50^{\circ} \mathrm{S}$ are considered.

\section{Results}

\subsection{Identification using winds}

The three different filters used to separate COLs from upper level troughs (see Fig. 1) are first analysed and discussed here. The relative vorticity is the preferred field for the tracking because more systems are identified using $\xi_{300}$ than $Z_{300}^{\prime}$. For a 36-year period (1979-2014), 540 tracks per year were identified using the single-point filter, 519 tracks with the four-point filter, and 300 tracks with the eight-point filter. Note that the numbers of the detected COLs using the singlepoint and the four-point filters are similar, but there is a significant reduction in number of identified COLs for the eight-point filter. This reduction is due to the greater number of constraints, i.e., the larger the restriction the smaller the sample size. 
A particular issue concerning the use of the stricter criteria (e.g. eight-point filter) is that the COL circulation varies through the lifecycle, resulting in problems of using fixed criteria. For example, we often find that many of the observed COLs present an elongated trough with significant westward tilt during the early stages of their lifecycle. This problem is illustrated in Fig. 2 for an individual COL in South America at two different stages. During the formation (Fig. 2a), the vorticity minimum identified by the method (represented by the symbol " $\mathrm{x}$ " in white color) is far offset from the COL centre due to the shear component associated with the upper-level flow, as discussed in Bell and Keyser (1993). On the other hand, objective methods work much better for COLs with more symmetric circulation which is more often found in the mature stage, as shown in Fig. 2b. The problem described above is minimised by using simpler criteria such as the one-point or four-point filters. This is a typical example of a problem that could be more accurately diagnosed using machine learning methods based on pattern recognition techniques. For example, such methods could offer the possibility to apply a filter that rotates in such a way that it matches the tilt starting from an initial set of rules, and then updating as more systems were detected.

The comparison between the three filtering methods is also performed by matching the same tracks as done in Hodges et al. (2011). The tracks are compared using a prescribed value for the mean separation distances, chosen here to be $4^{\circ}$ geodesic which overlaps in time by at least $50 \%$ of the track points. The largest absolute number of matches is observed between the single-point and the four-point filters (398 tracks per year), which corresponds to approximately $74 \%$ and $77 \%$ of the total tracks found in the single-point and the four-point filters, respectively. Comparing the single-point and the eight-point filters, there is a much lower number of matches (280 tracks per year), equivalent to $52 \%$ and $93 \%$ of the tracks found in the single-point and the eightpoint filters, respectively. Finally, the comparison between the four-point and the eight-point filters has 244 matches yearly, which corresponds to $47 \%$ and $81 \%$ of the tracks observed in the four-point and the eight-point filters, respectively. Surprisingly, the number of matches does not increase for the most intense COLs for each filter, as is the case for the comparison of COLs between different reanalyses (Pinheiro et al. 2019). This means the choice of the filtering method to detect cut-off lows does not affect the distribution of the COL intensities, i.e. the matches occur similarly for both strong and weak systems.

The comparison between the three filtering methods is also performed by analysing the maximum intensity distributions of the $300-\mathrm{hPa}$ COLs, determined by finding the $\xi_{300}$ maximum (scaled by -1 ) along the detected tracks for each filter, and shown in Fig. 3. The $\xi_{300}$ maxima are determined searching for the maximum value within $5^{\circ}$ geodesic radius centered on the $300-\mathrm{hPa}$ COL centre. It is apparent that there (a) Earlier Stage

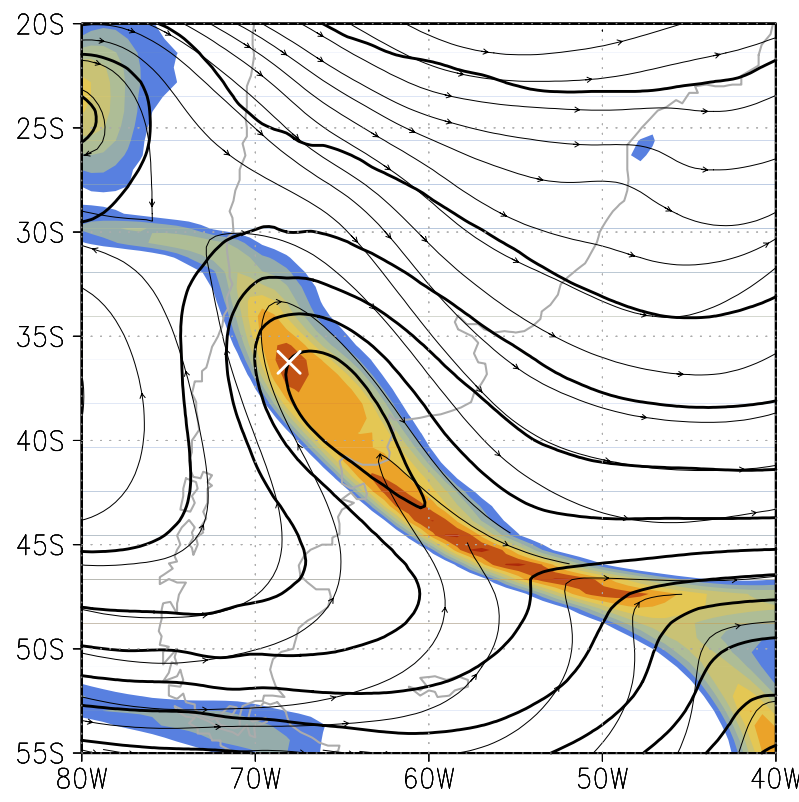

Fig. 2 An example of a 300-hPa Cut-off Low identified by the method for an a earlier stage (18 UTC 13 Apr 2010) and b later stage (18 UTC 15 Apr 2010) of the life cycle. Fields refer to the $300-\mathrm{hPa}$ level for geopotential height for interval contour of $50 \mathrm{gpm}$ (thick (b) Later Stage

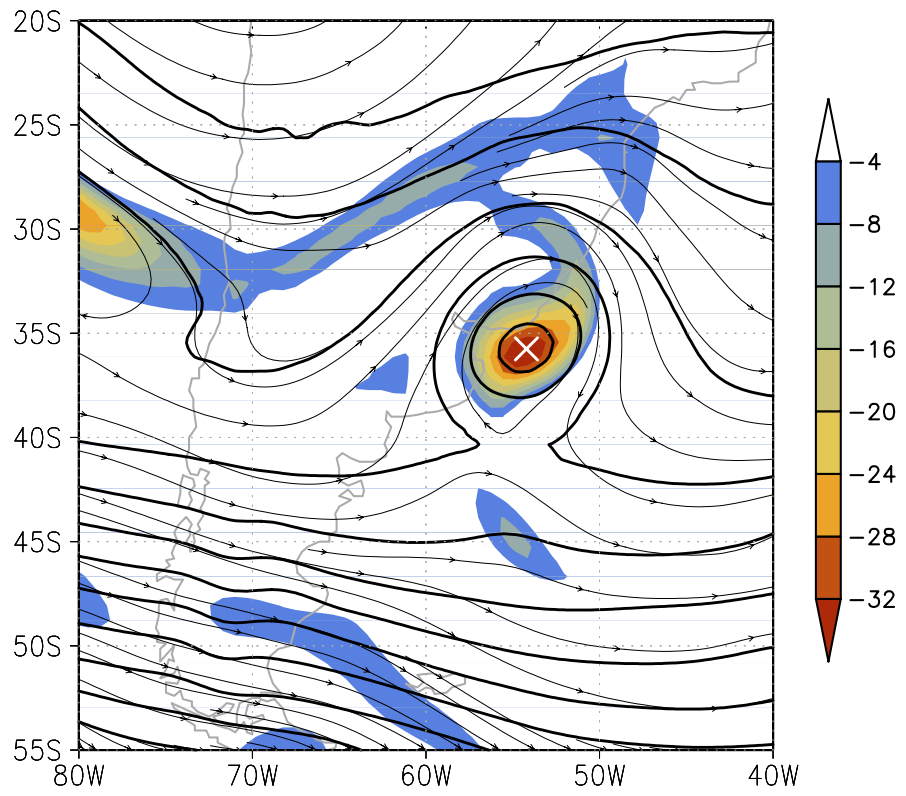

line), wind streamlines (thin line), and relative vorticity scale by $10^{-5}$ $\mathrm{s}^{-1}$ (shaded). The red color multiplication signs represent the minima identified by the objective detection 
(a)

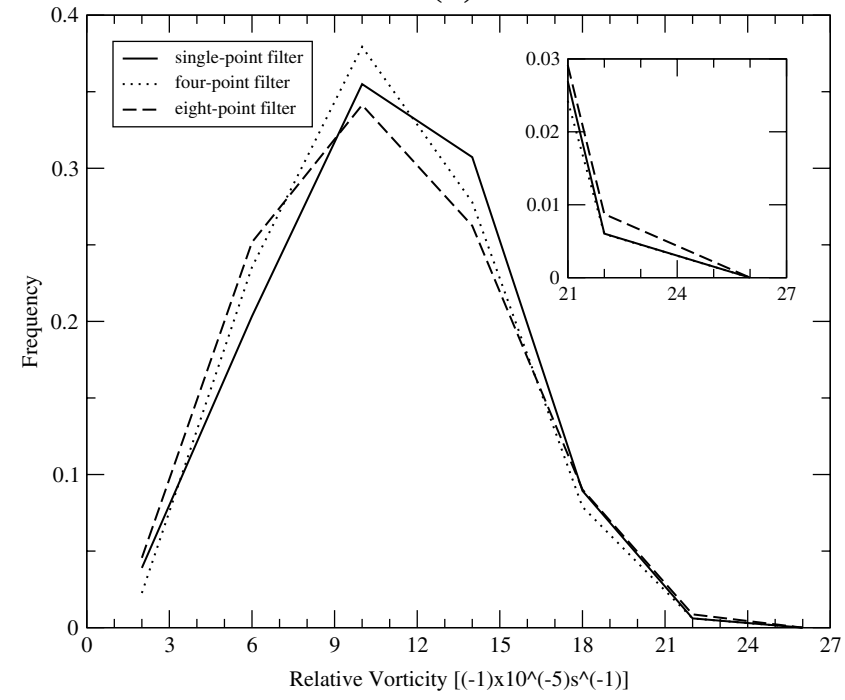

Fig. 3 a Frequency and b number distributions for the maximum intensity of the 300-hPa Cut-off Lows obtained with the single-point filter, four-point filter, and eight-point filter. Maximum intensity based on the $\xi_{300}$ scaled by -1 for the single-point filter (solid line),

is a similar intensity distribution for each of the filters, which have the maximum frequency and number for values around $10 \times 10^{-5} \mathrm{~s}^{-1}$. The extreme tails shown in the top inset graph in terms of frequency (Fig. 3a) show the tail is marginally longer in the eight-point filter than in other filters. However, the difference in number in respect of the extreme tails (Fig. 3b) is not significant among the filters. The conclusion is that although there are substantial differences in number, particularly with respect to the eight-point filter, the intensity distribution is similar for each filter. Therefore, the choice of the method to detect the cut-off circulation does not affect the type of detected system.

Though the results of this study provide quantitative analyses, the authors attempt to find which of the methods are as close as possible to "the ground truth" given the multiplechoice method derived from different variable combinations. An indication for the best method for our purpose was obtained after a visual inspection of the $Z_{300}$ maps (not shown) which shows that the highest number of COLs is observed for the single-point filter, followed in order by the four-point and eight-point filters. This means that the single-point filter detects more observed COLs than the other filters, but it also detects more unwanted systems, such as upper tropospheric troughs. We found that about $20 \%$ of the tracks identified with the single-point filter do not have an associated closed circulation, as observed visually in the geopotential maps. The method using the four-point filter, on the other hand, reduces the detection of unwanted tracks compared to the single-point filter, and it allows the detection of observed COLs with a number as large as observed in the method using the one-point (b)

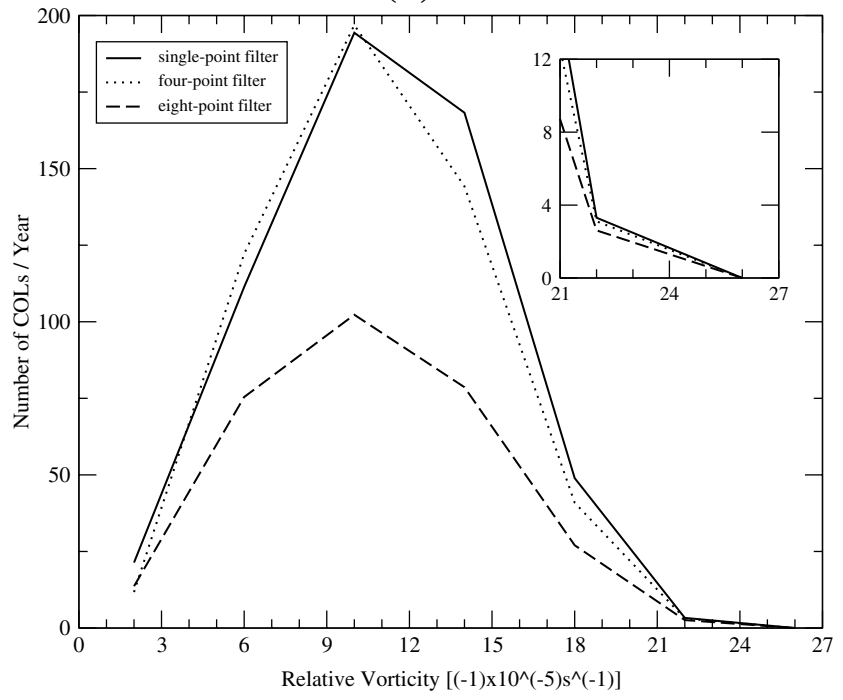

four-point filter (dotted line), and eight-point filter (dashed line). Analysis performed using the ERAI reanalysis for a 36-year period (1979-2014). The insets represent the high-intensity tails. Unit is s ${ }^{-1}$

filter. The eight-point filter retains fewer troughs than the other methods, but excludes many observed COLs. This happens, for example, when the axis of the COL tilts westward in the horizontal maps, as demonstrated in Fig. 2.

A particular observation is that the method detects longer tracks using relative vorticity than those observed in previous studies using geopotential, as the vorticity allows systems to be identified much earlier. These differences are minimised when using the cut-off stage instead of the full life cycle. The cut-off stage counts the time interval between the first and end time steps that satisfies the wind filter, for instance, the four-point filter. The full life cycle indicates the track in which the feature points satisfy the $\xi_{300}$ and $Z_{300}^{\prime}$ thresholds. By imposing the cut-off stage criterion, the distributions of COL lifetimes between $\xi_{300}$ and $Z_{300}^{\prime}$ are more similar than those observed in the full lifetime distributions. Figure 4 shows that most COLs last one or 2 days during the cut-off stage, although a few tracks persist for more than 10 days. This result is observed for both $\xi_{300}$ and $Z_{300}^{\prime}$ tracks. The mean lifetime of the cut-off stage is exactly the same for the $\xi_{300}$ and $Z_{300}^{\prime}$ COLs (2.9 days), similar to the mean lifetime found in previous studies using geopotential for the COL tracking (Fuenzalida et al. 2005; Campetella and Possia 2007; Reboita et al. 2010).

\subsection{Sensitivity of identifying Cut-off Lows using cold-core and stratospheric intrusion criteria}

The sensitivity of the method to identify COLs is also assessed with respect to different identification schemes in 


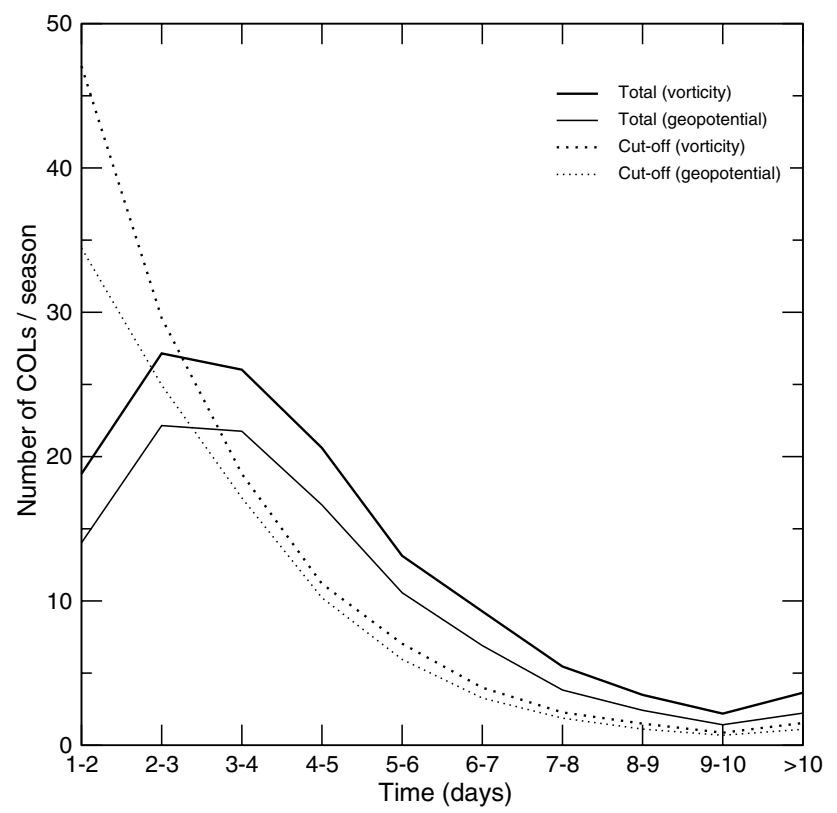

Fig. 4 Lifetime distribution in number for the total and cut-off stages identified by the $\xi_{300}$ and $Z_{300}^{\prime}$ Cut-off Lows in the Southern Hemisphere. Total duration in solid line, cut-off stage duration in dotted line. Vorticity in thick line, geopotential in thin line. Unit is days. Analysis performed using the ERAI reanalysis for a 36-year period (1979-2014)

(a)

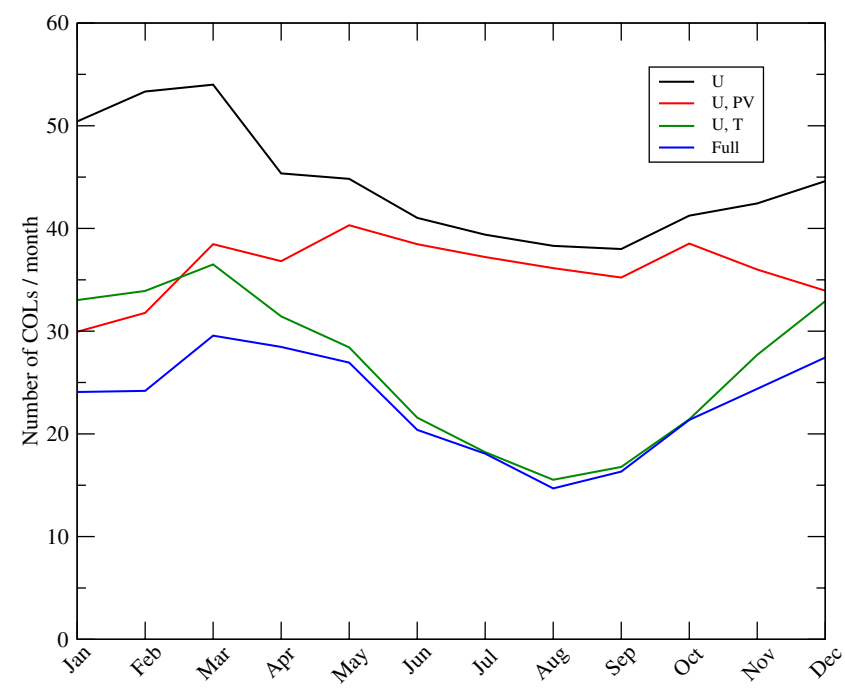

Fig. 5 a Monthly distribution, and $\mathbf{b}$ maximum intensity distribution (scaled by -1) of Cut-off Lows, obtained with the following criteria: (1) $U_{300}$; (2) $U_{300}$ and $P V_{300}$; (3) $U_{300}$ and $T_{300}$; (4) $U_{300}, P V_{300}$ and $T_{300}$ (Full). The four schemes for the $\xi_{300}$ Cut-off Lows are: (1) $U_{300}$ (black line); (2) $U_{300}$ and $P V_{300}$ (red line); (3) $U_{300}$ and $T_{300}$ (green line); (4) $U_{300}, P V_{300}$, and $T_{300}$ (blue line). The thresholds used in each variable terms of numbers, seasonality and intensity. The schemes use the $\xi_{300}$ for the tracking and the following additional fields: (1) $U_{300}$; (2) $U_{300}$ and $P V_{300}$; (3) $U_{300}$ and $T_{300}$; and (4) $U_{300}, P V_{300}$ and $T_{300}$. As expected, the largest number of COLs are found for the simplest methods (i.e. using only the $U_{300}$ ) with 540 tracks per year, whereas the smallest number of COLs identified is found for the multiple criteria methods (e.g. using $U_{300}, P V_{300}$, and $T_{300}$ ) with 285 tracks per year, i.e. the full set of criteria has about half the number of detected COLs compared to the simplest scheme. The schemes that use $U_{300} / P V_{300}$ and $U_{300} / T_{300}$ found 446 tracks and 327 tracks per year, respectively.

Figure 5a shows the monthly distribution and Fig. $5 b$ the intensity distribution (computed using the full resolution) of the detected COLs for the four different schemes. The single variable scheme, i.e. using only $U_{300}$, is used as a reference as this scheme detects the largest number of COLs observed in the $Z_{300}$ maps. For the single variable scheme compared with the full set of criteria (using $U_{300}, P V_{300}$ and $T_{300}$ ), the seasonality shows relatively small differences as both schemes show the maximum in March and the minimum in August or September. The frequency of the detected COLs differs between the two schemes, but the difference in number remains roughly constant through the year. However, the intensity distribution shows that the full set of criteria has more of the strongest systems than the method using a single parameter. For the comparison of the method using $T_{300}$ together with $U_{300}$, again using the single variable scheme

(b)

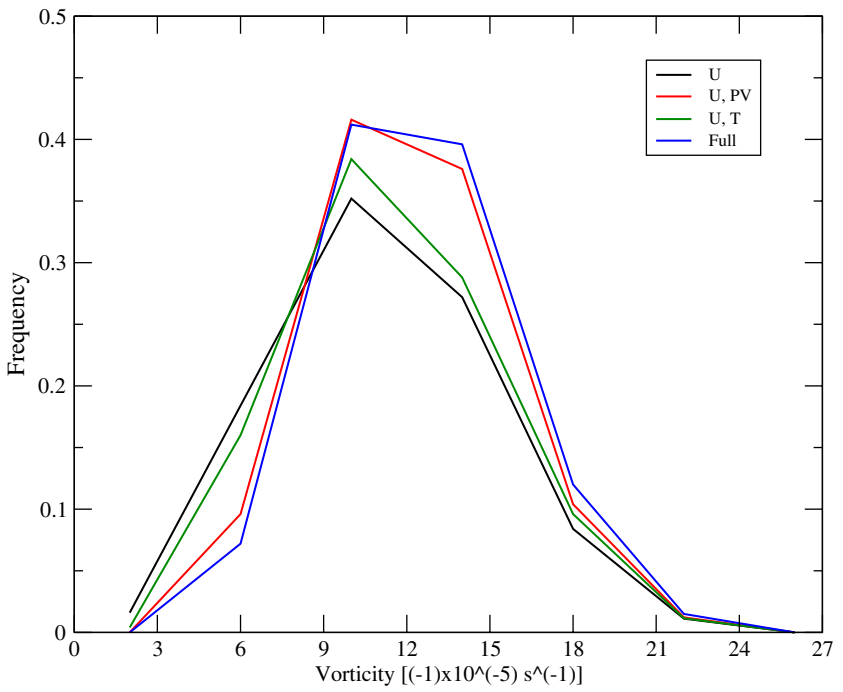

are: $U_{300}<-8.0 \mathrm{~m} / \mathrm{s}$; zonal anomaly of $T_{300}<-3.0^{\circ} \mathrm{C}$; zonal anomaly of $P V_{300}<-2.0$ PVU. The zonal anomalies of $P V_{300}$ and $T_{300}$ are determined searching for the minimum value within $5^{\circ}$ geodesic radius centered on the centre of Cut-off Lows. Analysis performed using the ERAI reanalysis for a 36-year period (1979-2014) 
as a reference, the largest differences are found in winter when typically the strongest COLs occur. Despite this difference, the seasonality and intensity distributions are similar between the two methods, suggesting that the type of the detected COLs does not change much when the cold-core criterion is used. Therefore, the use of the cold-core criterion seems to affect mainly the winter systems when the decrease in numbers occurs.

The largest difference in seasonality occurs when comparing the method using $P V_{300}$ together with $U_{300}$ as the maximum frequency is found in May, with a secondary maximum in October, and the minimum in January, indicating a somewhat similar distribution to that obtained for 500-hPa COLs (Fuenzalida et al. 2005; Ndarana and Waugh 2010). The monthly distributions show that the largest differences in number occur in the summer months, but it is relatively small for the period between May and October. The maximum frequency in winter may be as a result of the higher mean intensity of COLs (see Fig. 7), leading to a more pronounced tropopause folding as well as more efficient transport of cyclonic PV values from the stratosphere into the upper troposphere. In contrast, COLs are relatively weak in summer so that the PV intrusion is less effective compared to winter, reducing dramatically the number of detected COLs using the $U_{300} / P V_{300}$ method. Furthermore, the seasonal variation of the COL number appears to be related to the dynamical tropopause changes with season (Kunz et al. 2011) as the tropopause height reaches its lowermost (highermost) position in winter (summer), directly affecting the COL detection using schemes with PV, as previously demonstrated by Wernli and Sprenger (2007) and Ndarana and Waugh (2010). An alternative approach to reduce the seasonal variation of PV might be the use of temporal anomalies, but this would require an additional calculation.

Except for the scheme using $P V_{300}$ together with $U_{300}$, the other identification schemes have a similar seasonality of COLs as the peak and trough occurrence are found at a similar time of year, which is in agreement with previous studies (Ndarana and Waugh 2010; Favre et al. 2012), providing some confidence in how these systems are represented. However, even though the number of COLs identified does change, the number of COLs that occur in reality is unknown. Comparing these results with previous studies of COLs in the SH, Reboita et al. (2010) found substantial differences in frequency when using relatively old reanalyses (NCEP-NCAR and ERA-40) finding the annual average of the 300-hPa COLs is 197 using NCEP-NCAR and 349 using ERA-40. These numbers are much greater than the frequency shown by Ndarana and Waugh (2010) for a similar region for COLs at $250 \mathrm{hPa}$ (120 systems per year) using the NCEP-NCAR and a method to identify COLs similar to that used in Reboita et al. (2010), but with a more explicit imposition of the cold-core. The single variable scheme used in the present work identified an annual average of 547 COLs using the $\xi_{300}$ and 355 COLs using the $Z_{300}^{\prime}$ in ERAI. However, the frequency reduces to 285 COLs if using the full set of criteria for $\xi_{300}$, i.e. with cold-core and PV restrictions. According to Pinheiro et al. (2019), differences in number are also apparent between the modern reanalyses ERAI, NCEP Coupled Forecast System Reanalysis (NCEP CFSR), the second National Aeronautics and Space Administration Modern Era Retrospective Reanalysis for Research and Applications (NASA MERRA-2), and the Japanese 55-year Reanalysis (JRA-55), although there is a considerable improvement in the agreement between the new reanalyses compared to the older Japanese 25-year Reanalysis (JRA-25). These results confirm that the number of systems is very sensitive to the criteria and dataset used to identify COLs. It is also worth pointing out that it is difficult to fit the COLs into neat classes because the COLs by nature are more complicated than the typical aspects described in conceptual models.

\subsection{Spatial statistics for relative vorticity and geopotential COLs}

In this section, we present results for the comparison of the spatial statistics of COLs based on the relative vorticity and geopotential tracking and the four-point filter scheme (without PV and temperature restrictions), because this minimises issues associated with the increase (decrease) in number of identified troughs (COLs) compared to the other wind filters. The annual track densities for $\xi_{300}$ and $Z_{300}^{\prime}$ COLs in the SH are shown in Fig. 6. This shows that the $Z_{300}^{\prime}$ distribution is very similar to that obtained from the $\xi_{300}$ based on the same tracking methodology and selection criteria. The main areas of COLs are located near the continents for both $\xi_{300}$ and $Z_{300}^{\prime}$, but the density values differ substantially between the two fields. In general, the $\xi_{300}$ densities are greater than the $Z_{300}^{\prime}$ densities due to the larger number of COLs identified due to the differences in scales, as discussed in Hoskins and Hodges (2002). In addition to this aspect, there are other obvious differences between the $\xi_{300}$ and $Z_{300}^{\prime}$ spatial distributions. One is the $\xi_{300}$ track density maximum over the central Indian Ocean which is related to weak summer COLs (figure not shown). Another difference is that the $\xi_{300} \mathrm{COL}$ activity occurs at more northern latitudes in the SH than that observed for the $Z_{300}^{\prime}$, such as seen in northern Australia and South America. There are at least two reasons explaining the latitudinal differences. One is a result of the shear component effect that shifts the cyclonic vorticity maximum equatorward, possibly resulting in the differences of the track densities. The other factor may be due to the presence of stronger geopotential gradients and Laplacians as the COLs move poleward as a consequence of geostrophy. 


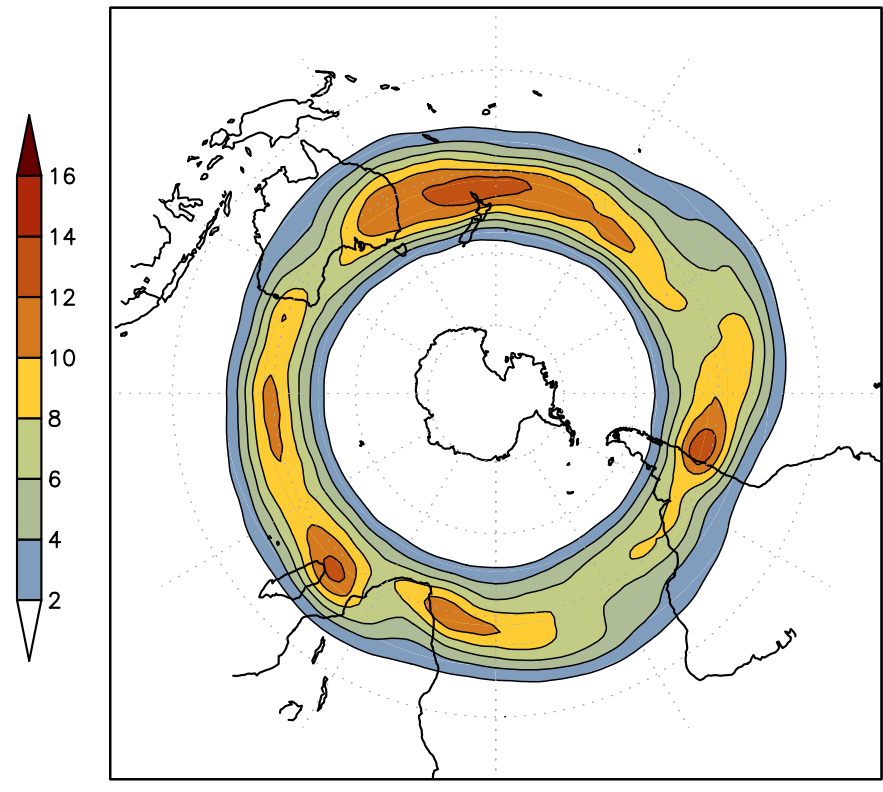

(a) 300-hPa Vorticity

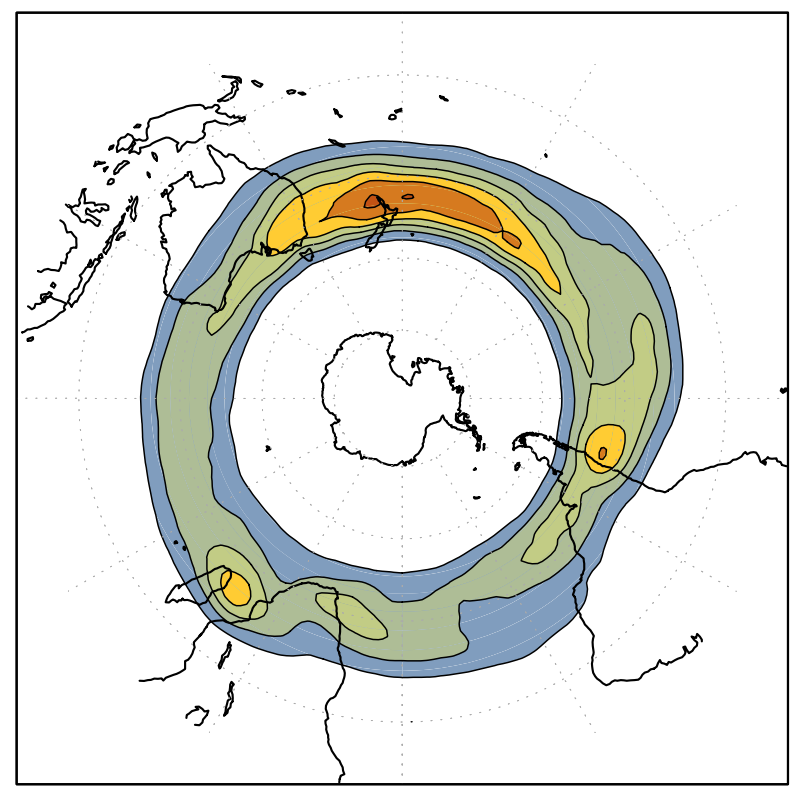

(b) $300-\mathrm{hPa}$ Geopotential
Fig. 6 Annual track density of Southern Hemisphere 300-hPa Cutoff Lows for a vorticity and $\mathbf{b}$ geopotential. Track density in shaded and solid line for contour interval of 2.0 units. Analysis is performed

Table 1 Number of $\xi_{300}$ and $Z_{300}^{\prime}$ Cut-off Lows, and the number of matches between the $\xi_{300}$ and $Z_{300}$

\begin{tabular}{lrrrrr}
\hline Period & MAM & JJA & \multicolumn{1}{c}{ SON } & \multicolumn{1}{c}{ DJF } & Annual \\
\hline Vorticity & 146.1 & 98.3 & 121.3 & 153.4 & 519.1 \\
Geopotential & 111.0 & 84.7 & 99.6 & 112.6 & 407.9 \\
Matches & 90.4 & 61.9 & 80.4 & 87.9 & 320.6 \\
\hline
\end{tabular}

The analysis is performed using the ERAI reanalysis for a 36-year period (1979-2014)

It is worthwhile mentioning that methods using fields that focus on the smaller synoptic scales (e.g. vorticity) tend to identify longer tracks than methods using larger-scale fields (e.g. geopotential). The choice of the tracking field affects the track density estimation since this statistic is sensitive to the track length. As a consequence longer tracks can result in higher track densities, and vice versa. This aspect may be the reason for the track density gaps in the ocean areas observed in the $Z_{300}^{\prime}$ compared to the $\xi_{300}$. A similar distribution of the $Z_{300}^{\prime}$ COLs has been shown in earlier studies based on the geopotential (Fuenzalida et al. 2005; Reboita et al. 2010).

Results for the number of $\xi_{300}$ and $Z_{300}^{\prime}$ COLs and the number of matches between the two sets for each season are shown in Table 1 . This shows that the annual average is 519.1 COLs for $\xi_{300}$ and 407.9 COLs for $Z_{300}^{\prime}$. The difference in number of COLs between the $\xi_{300}$ and $Z_{300}^{\prime}$ increases when no additional fields are used as criteria (figure not using the ERAI reanalysis for a 36-year period (1979-2014). Unit is number per season per unit area, the unit area is equivalent to a $5^{\circ}$ spherical cap $\left(\cong 10^{6} \mathrm{~km}^{2}\right)$

shown). This means the filter used to separate COLs from upper-level troughs (for instance, the four-point filter) makes the identification more stricter and reduces the spatial differences found between the relative vorticity and geopotential fields. Despite the differences in numbers, the seasonality is quite similar between the two fields, although it is somewhat more pronounced in $\xi_{300}$. The matches are determined for the tracks with a mean separation distance less than $4^{\circ}$ and an overlap in time greater than $50 \%$ of their points. The largest number of matches occurs in autumn with 90.4 tracks representing $81 \%(62 \%)$ of the $Z_{300}^{\prime}\left(\xi_{300}\right)$ tracks, while the lowest number of matches is observed in winter with 61.9 tracks representing $73 \%(63 \%)$ of the $Z_{300}^{\prime}\left(\xi_{300}\right)$ tracks. The reason for the relatively low number of matches in winter will be discussed later.

The seasonal intensity distribution of the $\xi_{300}$ and $Z_{300}^{\prime}$ COLs, computed from the full-resolution of $\xi_{300}$ and $Z_{300}^{\prime 300}$, is shown in Fig. 7. Values are determined from the maximum area averaged within a $5^{\circ}$ geodesic radius (scaled by -1), centered on the $\xi_{300}$ and $Z_{300}^{\prime}$ maxima. The probability density distribution for $\xi_{300}$ COLs, referenced to the $\xi_{300}$, provides a similar perspective to that shown in Pinheiro et al. (2017) in their Fig. 6 who used a multiple criteria scheme. The most intense $\xi_{300}$ COLs are found in winter, followed by spring, autumn and summer. Similarly the $Z_{300}^{\prime}$ distribution, referenced to the $Z_{300}^{\prime}$ tracks, also shows a broad range of values and significant differences between the seasons, with the strongest COLs occurring in winter. This last result 
(a) 300-hPa vorticity

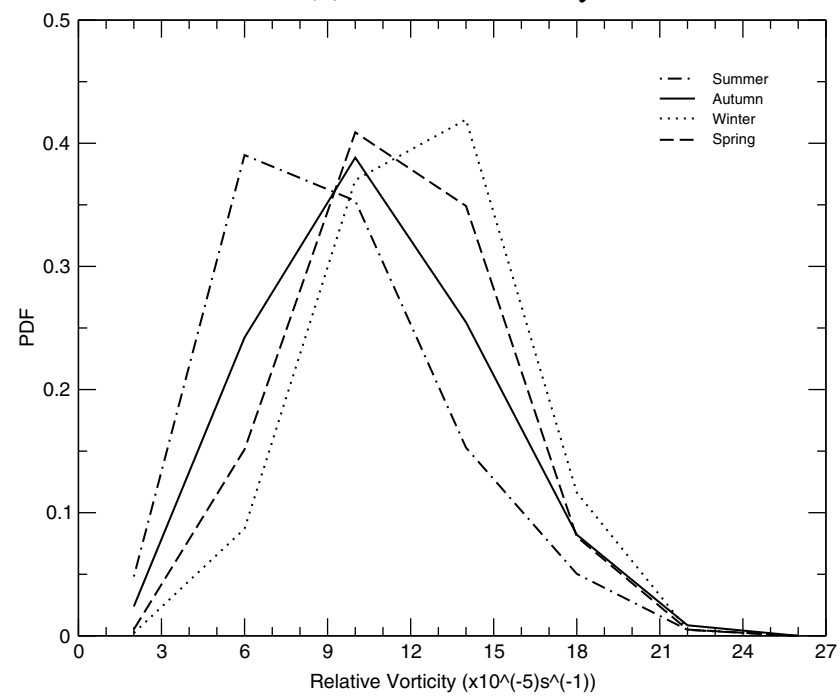

Fig. 7 Probability density function of the intensity for the $\mathbf{a} \xi_{300}$ and b $Z_{300}^{\prime}$ obtained for each austral season: summer (DJF, dashed-dotted line), autumn (MAM, solid line), winter (JJA, dotted line), and spring

contradicts what was observed by Fuenzalida et al. (2005) since these authors found no significant seasonal and geographical variations of intensity for 500-hPa COLs based on the Laplacian of 500-hPa geopotential. It is difficult to say what field is best at representing the intensities of COLs because vorticity and geopotential are very different.

To verify where the differences in the spatial distribution of the $\xi_{300}$ and $Z_{300}^{\prime}$ COLs occur, the track matching algorithm is used to construct statistics based on the tracks that match and do not match. Figure 8 shows the track density based on the tracks that match between the $\xi_{300}$ and $Z_{300}^{\prime}$, in white solid line, and the tracks that do not match, i.e., the difference between the $\xi_{300}$ and $Z_{300}^{\prime}$, in shaded. The results indicate that, in general, the regions of high density of matches coincide with the regions of high density of differences. This result is expected since the regions of matches and non-matches are in the same region as the main track density (see Fig. 6). This occurs for regions of high COL activity located around the main continental areas, in particular in summer and autumn when the density of matches and differences reach 12 and 10 per season (per unit area), respectively. High values for the matches and difference are also found over the oceans during the summer, such as over the Indian Ocean, mostly due to small-scale weak systems. Positive values for the density of difference means that in general there are more $\xi_{300}$ tracks than $Z_{300}^{\prime}$ tracks. A surprising opposite result occurs over southeastern Australia and parts of the Pacific in winter where the track density has larger values in $Z_{300}^{\prime}$ rather than in $\xi_{300}$. The reason for this result is not clear, (b) 300-hPa geopotential

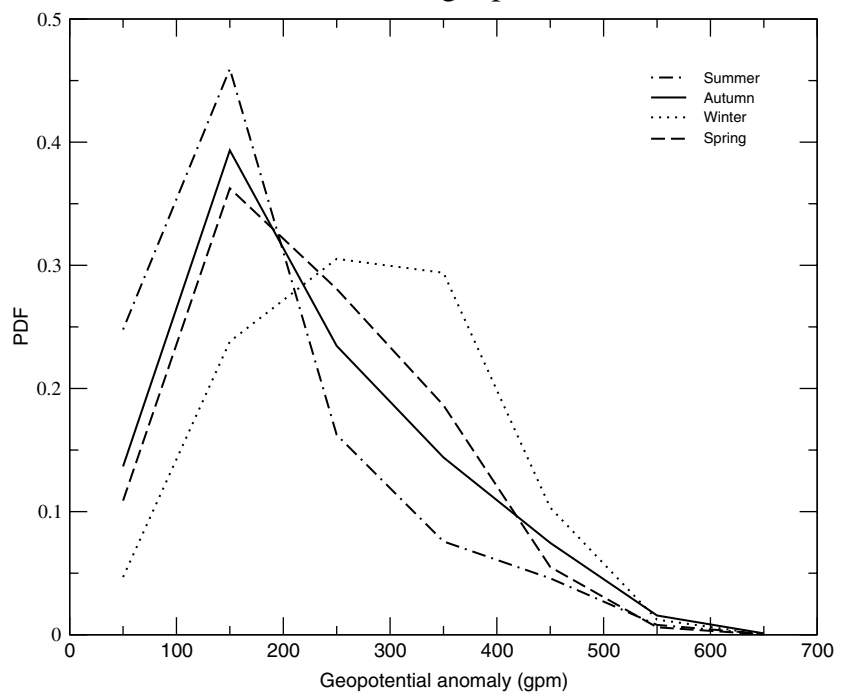

(SON, dashed line). Analysis performed for the period from 1979 to 2014. Units is $10^{-5} \mathrm{~s}^{-1}$ for vorticity and gpm for geopotential

but it may be explained by the fact that the $Z_{300}^{\prime}$ tracks are longer than the $\xi_{300}$ tracks in this region (figure not shown), and the longer tracks result in larger values of track density as discussed above. According to Pinheiro et al. (2017), the western Pacific is a preferred region for both COL genesis and lysis, and this aspect may result in uncertainties due to the difficult task of identifying the COL lifecycle. Similar problems are also found over the central Indian Ocean, where the track density values based on the matches are comparable to those based on the nonmatches. This occurs particularly during the winter, confirming the relatively small number of matches between the $\xi_{300}$ and $Z_{300}^{\prime}$ in this period, as shown in Table 1 .

To provide a more detailed understanding between relative vorticity and geopotential, a three-month period is used to give a view of how the tracks of COLs look between the two fields. Figure 9 shows the $\xi_{300}$ and $Z_{300}^{\prime}$ tracks plotted for the period of June, July and August 2010. The symbol ' $\mathrm{x}$ ' indicates the position of the genesis of each COL, and the colored lines indicate the intensity at each 6-hourly time step in units of $10^{-5} \mathrm{~s}^{-1}$ for relative vorticity, and in gpm for geopotential, both scaled by -1 . The most notable differences between the relative vorticity and geopotential are as follows: (1) the number of $\xi_{300}$ tracks is greater than the number of $Z_{300}^{\prime}$ tracks; (2) weaker systems are likely to be not identified for geopotential, this is the case for some tracks seen in southeastern Brazil and north of Australia; (3) the $\xi_{300}$ tracks are normally longer than the $Z_{300}^{\prime}$ tracks since the vorticity allows systems to be identified earlier in their life cycle. 


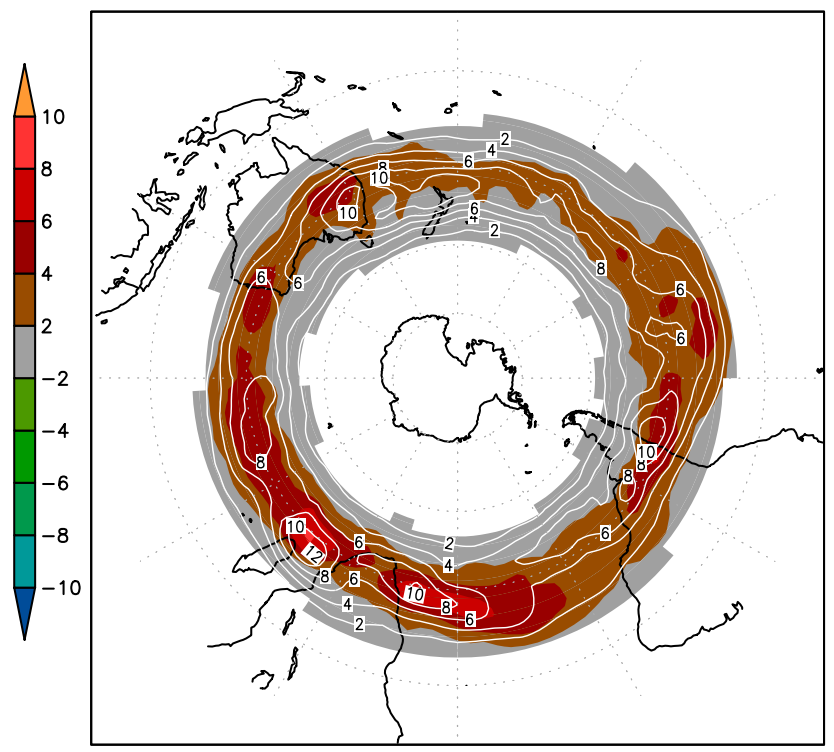

(a) Autumn

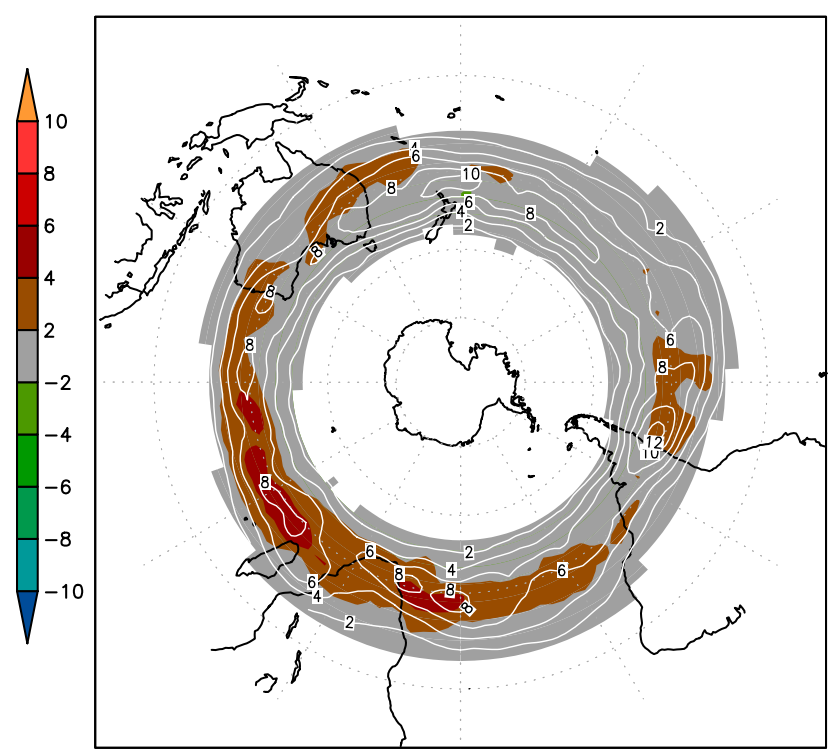

(c) Spring

Fig. 8 Seasonal track density of Cut-off Lows in the Southern Hemisphere for the matches and the difference between the $\xi_{300}$ and $Z_{300}^{\prime}$ tracks for a autumn (MAM), b) winter (JJA), c spring (SON), and $\mathbf{d}$ summer (DJF). Track density of matches between the $\xi_{300}$ and $Z_{300}^{\prime}$ tracks, represented in white solid line, and difference between the $\xi_{300}$ and $Z_{300}^{\prime}$ tracks, represented in shaded, both the contour intervals are

\section{Discussion and conclusions}

In the literature, there are a number of studies of COLs looking at different aspects and using varying identification methodologies, whose criteria differ widely, introducing uncertainties between the studies and their conclusions. The choice of a method to identify COL features is a difficult

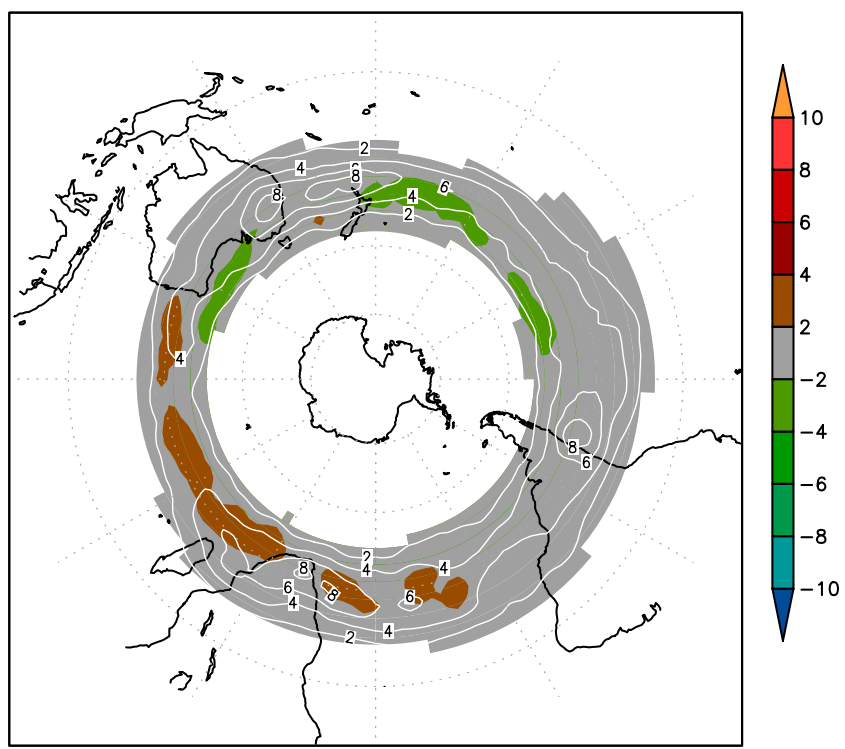

(b) Winter

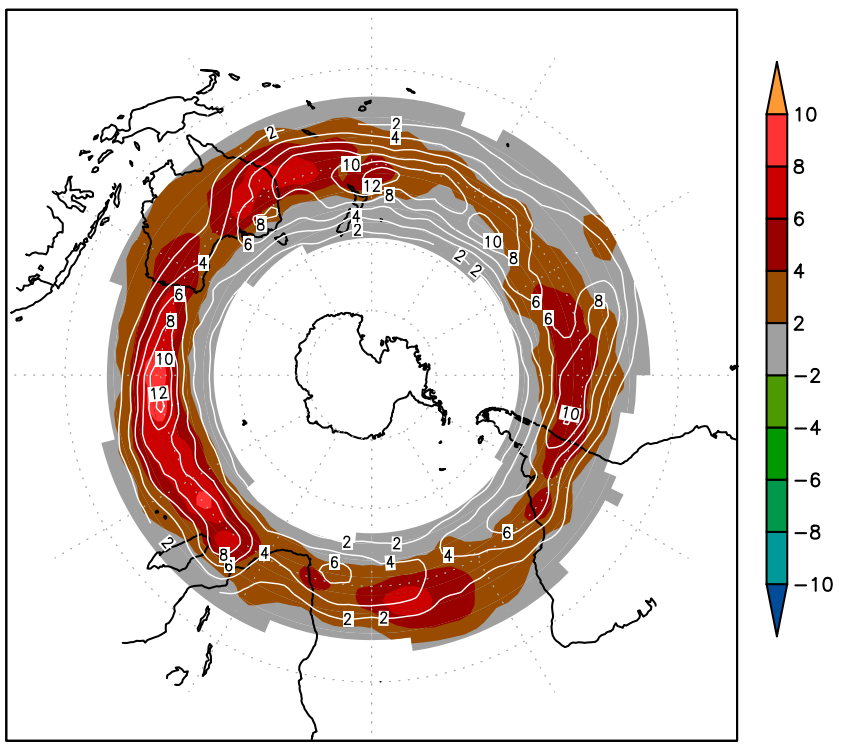

(d) Summer

2.0 units per season per unit area. The density of matches and difference have been suppressed were the vorticity track density is below 1.0 per season per unit area. The unit area is equivalent to a $5^{\circ}$ spherical cap $\left(\cong 10^{6} \mathrm{~km}^{2}\right)$. Analysis is performed using the ERAI for a 36-year period (1979-2014)

task, but it is a point that needs to be considered in order to yield consistent results and help in the COL forecasting, as the COLs can be complicated to follow in time. In this study, we have presented different methodologies that were employed in order to understand how different criteria affect numbers, seasonality and the intensity distribution of austral COLs. The study reported herein does not compare 
(a) 300-hPa vorticity

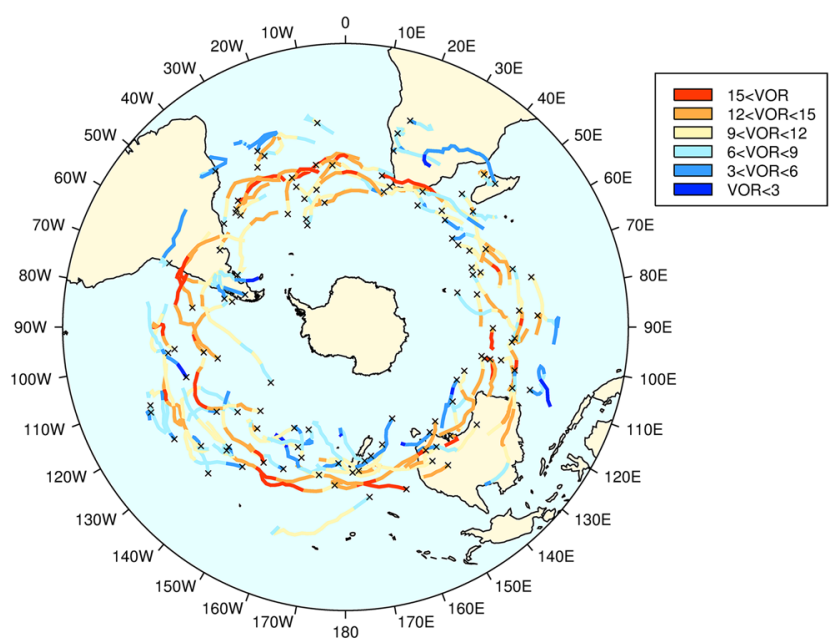

Fig. 9 Tracks of COLs in the Southern Hemisphere identified in a $\xi_{300}$ and $\mathbf{b} Z_{300}^{\prime}$. The symbol ' $\mathrm{x}$ ' indicates the position of the genesis of COL, the colored lines indicate the intensity at each 6-hourly

our results to those of other methodologies since the study of Pinheiro et al. (2017) has already made a comparison between previous studies. However, our results partially agree with the previous findings (e.g. Ndarana and Waugh 2010; Reboita et al. 2010) regarding the fact that the number and seasonality of COLs are dependent on the level used to identify the systems.Most studies using objective methods have restricted the tracking to relative vorticity, geopotential or geostrophic vorticity. This study has shown that by exploring a wide range of upper-level tropospheric fields, new perspectives can be obtained by identifying different features of 300-hPa COLs. The most obvious differences between using relative vorticity and geopotential to identify COLs is the larger number (length) of $\xi_{300}$ tracks compared to $Z_{300}^{\prime}$ tracks, and the fact that the weaker $\xi_{300}$ COLs are likely to be not identified using the $Z_{300}^{\prime}$. This is particularly the case for COLs at lower latitudes, where geopotential gradients are weaker than at higher latitudes. A similar argument can be applied to summer COLs which are typically weaker than winter COLs.

The comparison of COL identification methods has shown that the use of filtering is needed as a post-tracking step in order to separate COLs from other systems. Regarding the methods using only winds as additional requirements for the identification, the single and four-point filters give quantitatively similar results with the differences between each in general smaller than the differences found between the analysis using the eight-point filter. Despite the potential large differences in number of COLs, the derived intensity distribution is similar for each applied filter. Therefore, the choice of the method to detect the cut-off circulation does (b) 300-hPa geopotential

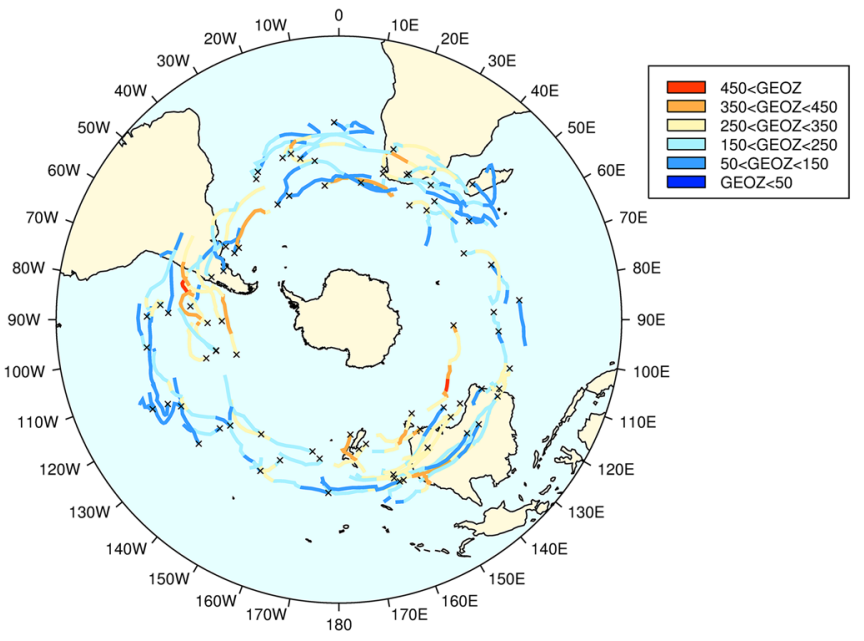

time step in units of $10^{-5} \mathrm{~s}^{-1}$ for vorticity and in geopotential meters for geopotential, both scaled by -1 . Analysis is performed using the ERAI reanalysis for the period from June to August 2010

not affect the type of detected system. The four-point filter in particular is considered the best compromise for the $\mathrm{COL}$ identification among the three types of filters (at least for the study region) because this minimises issues associated with the increase (decrease) in number of identified troughs (COLs). However, this of course involves a certain degree of subjectivity that reflects the complicated nature of COLs due to regional differences and the fact that the majority of these systems have short lifetimes imposing difficulties in defining a reference system.

The analysis of sensitivity in relation to specific variables used to identify COLs allows us to understand how the choice of method and threshold affect the results, and how these introduce uncertainties between studies due to the differences found in terms of seasonality and numbers. The four schemes (one scheme using only winds and three schemes using PV and/or temperature) used to identify COLs pick up distinct types of systems simply because different criteria impose different constraints. The single parameter scheme, which is based only on winds, was found to be less selective than the multiple step schemes, resulting in a larger number of either detected and observed COLs than other methods.

The sensitivity of the method with respect to PV indicates a good choice to detect the strongest systems, but it may be too restrictive in some cases, particularly for weak summer COLs. This result is likely related to how the spatial structure of PV varies with season. Since the PV anomalies associated with COLs can be from the result of the RWB process (Ndarana and Waugh 2010), which in turn is connected with the split jet flow (Berrisford et al. 2007), it is then plausible to assume that the seasonal variability of the 
jet stream affects the seasonality of COLs. In addition, the $\mathrm{COL}$ variations with season are not uniform over the $\mathrm{SH}$ due to the regional differences in the jet stream variability, since the shift of the jets throughout the year is greater in Australia and the southwestern Pacific than in other sectors (Archer and Caldeira 2008). This means that the SH COLs exhibit a regionally dependent seasonality, as observed in previous studies (Fuenzalida et al. 2005; Pinheiro et al. 2017).

The identification of a cold core as a condition to detect COLs is another aspect that leads to uncertainties between studies since the cold core search is generally performed at different layers, which seems to be chosen arbitrarily. In this study, the cold-core criterion is imposed at a fixed level (300-hPa), which makes the COL identification effective for most of situations. However, during the period of low activity of COLs (corresponding to winter and early spring) the tropopause in the low mid-latitudes is typically low when compared to other seasons (Appenzeller et al. 1996), leading to incursions of the stratospheric warm pool in the upperlevel COLs and, as a consequence, reducing the number of detected winter COLs compared to methods without a coldcore criterion. This is probably one of the main reasons why many studies found differences in the seasonality of COLs observed in similar regions.

Although it is not possible to say categorically which of these methods is the best choice for COL identification, due to the lack of a climatology that could be used as the reference, simpler schemes based only on winds (in our case the four-point filter) should be more representative of reality since they simply impose on the detection system a cyclonic circulation appearance regardless of the physical and dynamical characteristics. Therefore, this type of method may be considered as a standard method for identifying COLs that can be used for either operational or research purposes, providing some improvement particularly if we wish to identify large samples of COLs.

It is worth mentioning that the additional fields used in the method do not discriminate between COLs that are essentially formed at upper levels and those associated with frontal occlusions, because it is difficult to separate these using objective methods. In fact, there are no criteria to clearly distinguish the upper-level COLs from those originating with a frontal system since both of the two types of COLs often have similar characteristics, for example, a cold core in the middle upper troposphere (Pinheiro 2010). In addition, a characteristic of the conceptual model of Bell and Bosart (1989) is that the COLs are located equatorward of the main westerlies, but this is difficult to be implemented in algorithms, because in practice there is arbitrary choice in defining the jet stream position. Also, it is reasonable to suppose that the choice of method for COL identification may impact on the precipitation associated with COLs. These possibilities could be examined in further studies.
The differences from previous studies raise the question of whether the different conceptual models are due to regional differences in the COL structure. Thus, further study is needed to investigate the COL structure using a common set of analysis methods and applied to particular regions to see how methods would work better if one were able to detect regional and seasonal features. Finally, it is worth considering that, even if similar schemes were employed, the results could vary significantly depending on other aspects such as the level used to identify COLs, region, intrinsic variability, and uncertainties in datasets, such as reanalysis data (Pinheiro et al. 2019).

Acknowledgements The present study contains resultsfrom the thesis of the first author. CNPq (Conselho Nacional de Desenvolvimento Científico e Tecnológico) and CAPES (Coordenação de Aperfeiçoamento de Pessoal de Nível Superior) are acknowledged for the financial support for this work.

Open Access This article is distributed under the terms of the Creative Commons Attribution 4.0 International License (http://creativeco mmons.org/licenses/by/4.0/), which permits unrestricted use, distribution, and reproduction in any medium, provided you give appropriate credit to the original author(s) and the source, provide a link to the Creative Commons license, and indicate if changes were made.

\section{Appendix}

\section{Adaptive constraints for displacement distances and smoothness}

The upper-bound displacement $\left(d_{\max }\right)$ denotes the maximum allowed displacement in a time step in a specific zone generally defined for a latitudinal range, though this can also be applied with varying longitude regions, if required. For the purpose of this study, $d_{\max }$ was set at $3^{\circ}$. This threshold is much smaller than that used in Pinheiro et al. (2017) and is similar to those values generally used to track tropical storms which include depressions and cyclones. An example of $d_{\max }$ values used for a tropical application is given in Table 1 of Hodges (1999). Although the use of adaptive constraints helps to reduce the clutter due to the differences in features, there are some systems that do not satisfy the constraints, resulting in unrealistic or spurious tracking. If we make the $d_{\max }$ too large, larger changes in velocity would be allowed which could result in incorrect tracking. For example, using $d_{\max }=5^{\circ}$ we find some tracks in which a COL weakens at a certain time and intensifies in the next time step, leading to a new COL genesis from the same pre-existing upper-level trough, making the detected track longer than the observed COLs. This explains the larger mean lifetime for the detected $\xi_{300}$ COLs in Pinheiro et al. (2017) (7.3 days) compared to the detected $\xi_{300}$ COLs in the present study (4.1 days). The implementation of new constraints, which are found to be 
Table 2 Adaptive constraints used in Pinheiro et al. (2017)

\begin{tabular}{lrrrr}
\hline Zonal upper-bound displacements & & & \\
\hline Zones & \multicolumn{1}{l}{1} & \multicolumn{1}{l}{3} & \\
\hline Lower (degrees latitude) & -90.0 & -20.0 & 20.0 & \\
Upper (degrees latitude) & -20.0 & 20.0 & 90.0 & \\
$d_{\max }\left({ }^{\circ}\right)$ & 6.0 & 3.0 & 6.0 & \\
Adaptive track smoothness & & & & \\
$\bar{d}\left(^{\circ}\right)$ & 1.0 & 2.0 & 5.0 & $>8.0$ \\
$\psi_{\max }(\bar{d})$ & 1.0 & 0.3 & 0.1 & 0.01 \\
\hline
\end{tabular}

Table 3 Adaptive constraints used in the present study

\begin{tabular}{lrrrr}
\hline Zonal upper-bound displacements & & & \\
\hline Zones & \multicolumn{1}{l}{1} & \multicolumn{1}{l}{3} & \\
\hline Lower (degrees latitude) & -90.0 & -15.0 & 20.0 & \\
Upper (degrees latitude) & -15.0 & 20.0 & 90.0 & \\
$d_{\max }\left({ }^{\circ}\right)$ & 3.0 & 3.5 & 3.0 & \\
Adaptive track smoothness & & & & \\
$\bar{d}\left({ }^{\circ}\right)$ & 1.0 & 3.0 & 6.0 & $>8.0$ \\
$\Psi_{\max }(\bar{d})$ & 1.0 & 0.3 & 0.1 & 0.01 \\
\hline
\end{tabular}

more appropriate for the observed COL motion, makes the tracks closer to the typical observed tracks by excluding possibly more "merged systems" as well as more mobile earlier or later stages of the COL lifecycle.

The track smoothness constraint is measured in terms of changes in direction and speed. This is achieved by specifying values for the upper-bound track smoothness constraint $\left(\psi_{\max }\right)$ which is a function of the mean displacement distances over three time steps. The smoothness constraint is applied adaptively together with the displacement constraint, varying with the local mean separation distance on a track. The values used for the $\psi_{\max }$ and for the average displacement over three frames $(\bar{d})$ in each method are shown in Tables 2 and 3. These values are found to be suitable for the purpose of this study. The smoothness constraint is less restrictive at smaller distances between the track points (and vice versa). For slow-moving systems, for example, larger changes in velocity (speed and/or direction) are expected to occur in a time step in comparison to faster moving systems.

\section{References}

Ancellet G, Beekmann M, Papayiannis A (1994) Impact of a cut-off development on downward transport of ozone in the stratosphere. J Geophys Res 99:3451-3463

Appenzeller C, Holton JR, Rosenlof KH (1996) Seasonal variation of mass transport across the tropopause. J Geophys Res Atmos 101:15071-15078
Archer CL, Caldeira K (2008) Historical trends in the jet streams. Geophys Res Lett 35:L08803

Bell GD, Bosart LF (1989) A 15-year climatology of Northern Hemisphere $500 \mathrm{mb}$ closed cyclone and anticyclone centers. Mon Weather Rev 117:2142-2164

Bell GD, Keyser D (1993) Shear and curvature vorticity and potential vorticity interchanges: interpretation and application to a cut-off cyclone event. Mon Weather Rev 121:76-102

Berrisford P, Hoskins BJ, Tyrlis E (2007) Blocking and Rossby wave breaking on the dynamical tropopause in the Southern Hemisphere. J Atmos Sci 64:2881-2898

Campetella C, Possia N (2007) Upper-level cut-off lows in southern South America. Meteorol Atmos Phys 96:181-191

Costa SB (2009) Balanço de vorticidade e energia aplicados aos vórtices ciclônicos de altos níveis atuantes no Oceano Atlântico Tropical Sul e adjacências. Dissertation, Universidade de São Paulo

Dee DP et al (2011) The ERAI reanalysis: configuration and performance of the data assimilation system. Q J R Meteorol Soc 137:553-597

Favre A, Hewitson B, Tadross M, Lennard C, Cerezo-Mota R (2012) Relationships between cut-off lows and the semiannual and southern oscillations. Clim Dyn 38:1473-1487

Fuenzalida H, Sánchez R, Garreaud R (2005) A climatology of cutoff lows in the Southern Hemisphere. J Geophys Res 110:1-10

Hodges KI (1994) A general Method for tracking analysis and its application to meteorological data. Am Meteorol Soc 122:2573-2585

Hodges KU (1995) Feature tracking on the unit sphere. Mon Weather Rev 123:3458-3465

Hodges KI (1996) Spherical nonparametric estimators applied to the UGAMP model integration for AMIP. Mon Weather Rev 124:2914-2932

Hodges KI (1999) Adaptive constraints for feature tracking. Mon Weather Rev 127:1362-1373

Hodges KI, Lee RW, Bengtsson L (2011) A comparison of extratropical cyclones in recent reanalyses ERAI, NASA MERRA, NCEP CFSR, and JRA-25. J Clim 24:4888-4906

Holton JR (1992) An introduction to dynamic meteorology, 4th edn. Academi Press, Walthan

Hoskins JB, Hodges KI (2002) New perspectives on the Northern Hemisphere winter storm tracks. J Atmos Sci 59:1041-1061

Hoskins JB, Hodges KI (2005) A new perspective on the Northern Hemisphere winter storms tracks. J Clim 18:4108-4129

Hoskins BJ, Sardeshmukh PD (1984) Spectral smoothing on the sphere. Mon Weather Rev 112:2524-2529

Hoskins BJ, Mcintyre ME, Robertson AW (1985) On the use and significance of isentropic potential vorticity maps. Q J R Meteorol Soc 111:877-946

Kentarchos AS, Davis TD (1998) A climatology of cut-off lows at $200 \mathrm{hPa}$ in the Northern Hemisphere, 1990-1994. Int J Climatol 18:379-390

Kunz A, Konopka P, Müller R, Pan LL (2011) Dynamical tropopause based on isentropic potential vorticity gradients. J Geophys Res Atmos 116:D1

Llasat MC, Martín F, Barrera A (2007) From the concept of "Kaltlufttropfen" (cold air pool) to the cut-off low. The case of September 1971 in Spain as an example of their role in heavy rainfalls. Meteorol Atmos Phys 96:43-60

McInnes KL, Hubbert GD (2001) The impact of eastern Australian cut-off lows on coastal sea levels. Meteorol Appl 8:229-244

Morais MDC (2016) Vórtices ciclônicos de altos níveis que atuam no bordeste do Brasil. Dissertation, Instituto Nacional de Pesquisas Espaciais

Ndarana T, Waugh DW (2010) The link between cut-off lows and Rossby wave breaking in the Southern Hemisphere. Q J R Meteorol Soc 136:869-885 
Nieto R, Gimeno L, de la Torre L, Ribeira P, Gallego D, García-Herrera R, García JA, Nuñez M, Redãno A, Lorente J (2005) Climatological features of cutoff low systems in the Northern Hemisphere. J Clim 18:3085-3103

Nieto R, Sprenger M, Wernli H, Trigo RM, Gimeno L (2008) Identification and climatology of cut-off low near the tropopause. Ann N Y Acad Sci 1146:256-290

Pinheiro HR (2010) Objective identification of upper tropospheric cutoff lows in subtropical regions. Dissertation, National Institute for Space Research

Pinheiro HR, Hodges KI, Gan MA, Ferreira NJ (2017) A new perspective of the climatological features of upper-level cut-off lows in the Southern Hemisphere. Clim Dyn 48:541-559

Pinheiro HR, Hodges KI, Gan MA (2019) An intercomparion of subtropical Cut-off Lows in the Southern Hemisphere using recent reanalyses: ERA-Interim, NCEP-CFSR, MERRA-2, JRA-55, and JRA-25. Clim Dyn (submitted)

Price JD, Vaughan G (1992) Statistical studies of cut-off low systems. Ann Geophys 10:96-102

Reboita MS, Nieto R, Gimeno L, Rocha RP, Ambrizzi T, Garreaud R, Kruger LF (2010) Climatological features of cutoff low systems in the Southern Hemisphere. J Geophys Res Atmos 115:D17104
Rondanelli R, Gallardo L, Garreaud RD (2002) Rapid changes in ozone mixing ratios at Cerro Tololo $\left(30^{\circ} 10^{\circ} \mathrm{S}, 70^{\circ} 48^{\circ} \mathrm{W}, 2200 \mathrm{~m}\right)$ in connection with cut-off lows and deep troughs. J Geophys 107:D23

Simmons A, Uppala S, Dee D, Kobayashi S (2007) ERA interim: new ECMWF reanalysis products from 1989 onwards. ECMWF Newslett 110:25-35

Singleton AT, Reason CJC (2006) A Numerical model study of an intense cutoff low pressure system over South Africa. Mon Weather Rev 135:1128-1150

Taljaard JJ (1985) Cut-off lows in the South African region. S Afr Weather Bureau Tech Paper 14:154

Wernli H, Sprenger M (2007) Identification and ERA-15 climatology of potential vorticity streamers and cutoffs near the extratropical tropopause. J Atmos Sci 64:1569-1586

Publisher's Note Springer Nature remains neutral with regard to jurisdictional claims in published maps and institutional affiliations. 